\title{
Use of DNAzymes for cancer research and therapy
}

\author{
XU ZhiJie ${ }^{1,2}$, YANG LiFang ${ }^{1,2}$, SUN LunQuan ${ }^{2} \&$ CAO Ya ${ }^{1,2^{*}}$ \\ ${ }^{1}$ Key Laboratory of Carcinogenesis of Chinese Ministry of Public Health, Key Laboratory of Carcinogenesis and Cancer Invasion of Chinese \\ Ministry of Education, Cancer Research Institute, Xiangya School of Medicine, Central South University, Changsha 410008, China; \\ ${ }^{2}$ Center for Molecular Medicine, Xiangya Hospital, Central South University, Changsha 410008, China
}

Received January 15, 2012; accepted March 1, 2012

\begin{abstract}
DNAzymes (Dzs) are single-stranded DNA catalysts that specifically cleave the mRNA of targeted genes. Compared with other gene-silencing technologies, such as ribozymes, antisense oligonucleotide and small interference RNA (siRNA), DNAzymes have several advantages, including small molecular weight, diversity, low cost and relative stability in serum. With the evolution of molecular technology, the first DNAzyme was generated in vitro in 1994. From then on, DNAzymes have been studied in order to understand their structures, chemistry and biological applications. Particularly, DNAzymes have been widely applied as a new interference strategy in the treatment of many conditions, including cancer, viral diseases, and cardiovascular diseases. This review mainly summarizes the use of DNAzymes in the areas of cancer research and therapy.
\end{abstract}

DNAzymes, gene-silencing technologies, interference strategy, cancer

Citation: $\quad$ Xu Z J, Yang L F, Sun L Q, et al. Use of DNAzymes for cancer research and therapy. Chin Sci Bull, 2012, 57: 3404-3408, doi: 10.1007/s11434$012-5380-\mathrm{Z}$

The first DNAzyme was produced experimentally in 1994 by Breaker and Joyce [1], who used in vitro selection to identify a special DNA sequence that catalyzes $\mathrm{Pb}^{2+}$-dependent cleavage of an RNA phosphodiester linkage. Afterwards, a number of DNAzymes were created with the capacity to catalyze many reactions, including the cleavage of DNA or RNA, the modification and ligation of DNA, and the metalation of porphyrin rings. The catalytic properties of DNAzymes have been utilized in therapeutic and biosensor applications.

The most commonly studied RNA-cleaving DNAzymes are 10-23 (Figure 1(a)) and 8-17 (Figure 1(b)), which both require metal ions such as $\mathrm{Mg}^{2+}$ for their activity [2]. The 8-17 DNAzyme appears to be the simplest DNA motif capable of RNA cleavage while the 10-23 DNAzyme is the best understood model. Each 10-23 DNAzyme has a catalytic domain of 15 deoxyribonucleotides flanked by 2 arms, each composed of a substrate-recognition domain of 7-9 deoxyribonucleotides. This DNAzyme effectively cleaves

*Corresponding author (email: ycao98@ vip.sina.com) the substrate RNA at unpaired purine: paired pyrimidine junctions under physiological conditions [3]. Additional RNA-cleaving DNAzymes have also been identified: some DNAzymes do not need a divalent metal ion cofactor (Figure $1(\mathrm{c}))$ and others that require the amino acid histidine as an obligatory cofactor (Figure 1(d)) [2].

DNAzymes have the advantages of small molecular (a)

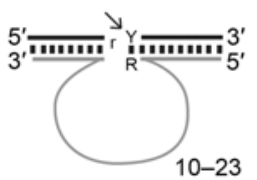

(c)

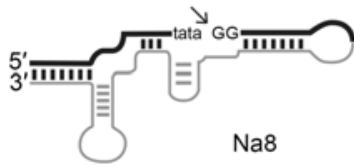

(b)

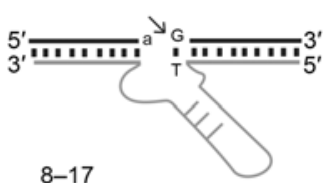

(d)

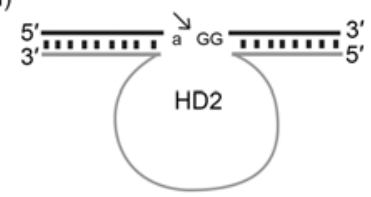

Figure 1 The structure of DNAzymes. Black lines denote RNA; gray lines denote DNAzyme. $r$ and R, purine; Y, pyrimidine. 
weight, low cost and relative serum stability. However, there are also some disadvantages such as off-target effects. For example, Dz13, a DNAzyme that cleaves $c$-Jun mRNA, is effective against tumors directly, and it also induces a cytotoxic stress response. This off-target effect may be induced by up-regulation of the tumor suppressor E2F1, which occurs regardless of cellular $p 53$ status [4]. Table 1 provides a summary of the advantages and disadvantages of DNAzymes and three other common gene intervention techniques.

\section{Applications of DNAzymes in cancer studies}

The DNAzymes widely used in cancer research are shown in Table 2. Yang et al. [7] suggested that a $L M P 1$-specific DNAzyme down-regulates the expression of the EBV oncoprotein LMP1 and inhibits the cellular signaling pathways activated by LMP1. This group used a cationic porphyrin TMP as a transfection reagent. To increase stability of the DNAzyme in cells, two phosphorothioate (PS) linkages were introduced to both ends of the arms [7,8]. When an egr-1-targeted DNAzyme, another DNAzyme used in cancer research, was synthesized with an inverted thymidine (Ti) at the $3^{\prime}$ position, the DNAzyme could provide efficient resistance to nucleolytic degradation [9].

\subsection{DNAzymes inhibit cell proliferation}

The $b c r-a b l$ oncogene, also known as the Philadelphia chromosome $(\mathrm{Ph})$, is detected in $>95 \%$ of patients with chronic myelogenous leukemia (CML) and in $20 \%-30 \%$ of adults with acute lymphoblastic leukemia (ALL). The BCRABL fusion proteins, encoded by the $b c r$-abl oncogene, inhibit apoptosis and induce the accumulation of myeloid cells. Among these fusion proteins, p210 $0^{\text {bcr-abl }}$ and p190 bcr-abl both have increased tyrosine kinase enzymatic activity. The over-expression of the kinase is thought to confer a growth advantage to $\mathrm{Ph}^{+} \mathrm{CML}$ or ALL cells and to play a momentous role in the pathogenesis of the malignancies. An elegant study demonstrated that the DNAzymes targeting $b c r$ - $a b l$ efficiently cleave only their respective $b c r-a b l$ substrates, which led to down-regulation of $\mathrm{p} 210^{\mathrm{bcr}-\mathrm{abl}}$ protein expression and significant inhibition of the proliferation of $\mathrm{Ph}^{+}$malignant cells. These $b c r$ - $a b l$-specific DNAzymes may improve the success rate of autologous stem cell transplants by purging the leukemia stem cell populations from patients with $\mathrm{Ph}^{+}$leukemia [19].

$P M L / R A R \alpha$ fusion transcripts are detected in all patients with the $\mathrm{t}(15: 17)$ translocation, which fuses the retinoic acid receptor $\alpha(R A R \alpha)$ gene on chromosome 17 to the promyelocytic leukaemia $(P M L)$ gene on chromosome 15 to produce the $P M L / R A R \alpha$ gene. These transcripts are critical

Table 1 Advantages and disadvantages of DNAzymes compared with ribozymes, antisense oligonucleotide (ASO) and small interference RNA (siRNA)

\begin{tabular}{|c|c|c|c|}
\hline Gene intervention techniques & Advantages & Disadvantages & References \\
\hline DNAzymes & More stable; easier to synthesize; less expensive & Off-target effects & {$[2,4]$} \\
\hline Ribozymes & Present in nature & Less stable; toxic & {$[5]$} \\
\hline ASO & Not dependent on cofactor & Less sensible; less selective & {$[5]$} \\
\hline siRNA & More active; not dependent on mRNA sequences & $\begin{array}{l}\text { Dependent on cellular mechanisms; less specific; immune } \\
\text { response and off-target effects }\end{array}$ & {$[6]$} \\
\hline
\end{tabular}

Table 2 Applications of DNAzymes in cancer research

\begin{tabular}{|c|c|c|c|}
\hline Cancer & Target & Biological effects (in vitro and in vivo) & References \\
\hline Nasopharyngeal carcinoma & $L M P 1$ & Inhibiting proliferation and metastasis; promoting apoptosis; enhancing radiosensitivity & {$[7,8]$} \\
\hline Breast cancer & egr-1 & Inhibiting proliferation and metastasis; suppressing tumor growth & [9] \\
\hline Hepatocarcinoma & $I G F-I I$ & Inhibiting adhesion, invasion and metastasis & {$[12]$} \\
\hline Pancreatic carcinoma & Survivin & Inhibiting proliferation; promoting apoptosis & [13] \\
\hline \multirow[t]{3}{*}{ Colorectal cancer } & $\beta 1$-integrin & Inhibiting adhesion and invasion & [14] \\
\hline & $\beta$-catenin & Inhibiting proliferation & [15] \\
\hline & $k$-ras & Enhancing radiosensitivity & {$[16]$} \\
\hline Prostatic cancer & Aurora kinase A & Inhibiting proliferation and metastasis; promoting apoptosis; down-regulating telomerase activity & [17] \\
\hline Epithelioma & c-Jun & Inhibiting proliferation; blocking angiogenesis; suppressing tumor growth & [18] \\
\hline \multirow[t]{2}{*}{ Leukocythemia } & Bcr-abl & Inhibiting proliferation; enhancing success rate of autologous stem cell transplants & [19] \\
\hline & $P M L / R A R \alpha$ & Inhibiting proliferation and vitality; promoting apoptosis & {$[20]$} \\
\hline \multirow[t]{2}{*}{ Osteosarcoma } & $u P A R$ & Inhibiting invasion & {$[22]$} \\
\hline & Ezrin & Inhibiting proliferation & [23] \\
\hline
\end{tabular}


in the development of acute promyelocytic leukemia (APL). DNAzymes that cleave the $P M L / R A R \alpha$ transcripts were designed by Kabuli et al. [20]. This research indicated that these DNAzymes cleave PML/RAR $\alpha$ efficiently and specifically at the level of mRNA and protein. Moreover, these DNAzymes not only show a statistically significant suppression of cell proliferation and viability, but also promote apoptosis of the cells. These biological effects of DNAzymes can be enhanced when combined with all-trans-retinoic acid (ATRA) [20].

\subsection{DNAzymes promote cell apoptosis}

Defective apoptosis has been shown to play a critical role in tumorigenesis. Apoptosis is mediated by caspases and two main apoptosis regulator families: the Bcl-2 family and the inhibitor of apoptosis proteins (IAP). Survivin, identified recently, is the most structurally unique member of the IAP family and functions in controlling cell division and the inhibition of apoptosis. As survivin is expressed in most human tumors but rarely in terminally differentiated normal tissues, its expression follows a tumor-specific pattern. Liang et al. [13] suggested that a DNAzyme targeting survivin mRNA markedly increases apoptosis and inhibits the growth of human pancreatic carcinoma cell lines. The results also showed that the survivin-targeted DNAzyme can block the cells from entering into $\mathrm{S}$ phase and passing through the G2/M checkpoint.

It is well known that caspases play an important role in apoptosis. Recently, it has been reported that loss of caspase2 , one member of the caspases family, can lead to malignancy, suggesting that caspase- 2 is a tumor suppressor. However, the mechanism of this biological effect is still unknown. In one recent study, Dass et al. [24] found that Dz13, a DNAzyme that cleaves $c$-Jun mRNA, induces activation of the caspase-2 protein rather than up-regulating the gene's activity, which results in cells exhibiting signs of late apoptosis. These results also confirmed the off-target effects of Dz13 in cancer cells.

\subsection{DNAzymes suppress cell adhesion and invasion}

Local invasion of the host tissue and metastasis are hallmarks of cancer progression, and understanding cancer cell motility is essential for therapeutic targeting of cancer [25]. It is already known that integrins play a primary role in the motility of cancer cells and that the expression levels of numerous integrins can be up-regulated during cancer progression. Among these integrins, the $\beta 1$-integrin subfamily plays a crucial role in the early stages of angiogenesis and in the invasiveness of cancer cell lines. A recent study provided direct evidence that blocking the synthesis of the $\beta 1$-integrin subunit using a specific DNAzyme inhibits the adhesion and invasion of carcinoma cell lines [14]. Stec et al. [26] further suggested that DNAzymes targeting $\beta 1$ 1053 and $\beta 3-1243$ markedly inhibit expression of $\beta 1$ and $\beta 3$ integrin subunits at the level of mRNA and protein, and specifically decrease the cell surface expression of corresponding subunits in endothelial cells. In addition, the adhesion, invasion and metastasis of endothelial cells are all significantly blocked by the DNAzymes to $\beta 1$ and $\beta 3$ mRNA.

Cancer cells can metastasize only if they penetrate the extracellular matrix (ECM) and the basement membrane. Urokinase-type plasminogen activator (uPA) receptor (uPAR) has been implicated in signal transduction and biological processes such as cancer metastasis, angiogenesis and cell migration. When bound to its receptor, uPA has the ability to trigger proteolysis cascades that, depending on the physiological environment, participate in the ECM and basement membrane degradation, activate latent transforming growth factor- $\beta$ (TGF- $\beta$ ), release basic fibroblast growth factor (FGF) or activate zymogens of matrix metalloproteinases (MMPs). It is believed that the tissue degradation following plasminogen activation facilitates tissue invasion by cancer cells and, thus, contributes to metastasis. de Bock et al. [22] indicated that the $U P A R$-targeted DNAzymes, Dz483 and Dz720, cleave $u P A R$ transcripts in vitro with high efficacy and specificity at a molar ratio of $U P A R$ to $\mathrm{Dz}$ as low as $1: 0.2$. The decrease of $u P A R$ expression significantly suppresses cell invasion and metastasis. The optimum doses of DNAzyme are in the range of 1.6-3.2 $\mu \mathrm{g} / \mathrm{mL}$.

It has been well recognized that growth and metastasis of solid tumors require continuous angiogenesis. The VEGF sub-family of growth factors contains important signaling proteins involved in the activation and inhibition of angiogenesis. A DNAzyme targeting VEGFR-2, one cognate receptor of VEGF, is found to cleave its substrate efficiently, and thus, inhibit the proliferation of endothelial cells and tumor growth in vivo [27]. In addition, Dz13 also significantly inhibits endothelial cell proliferation, migration, chemoinvasion and tubule formation, which suppressed the growth of solid tumors and angiogenesis [28].

\subsection{DNAzymes enhance cell radiosensitivity}

It has been established that EBV infection is associated with the occurrence and development of nasopharyngeal carcinoma (NPC). Latent membrane 1 (LMP1) is an important viral protein associated with Epstein-Barr virus that has some oncogenic properties during latent infection and may lead to NPC. A DNAzyme targeting the LMPl mRNA, Dzl, significantly down-regulates the expression of $L M P 1$ through NF$\kappa \mathrm{B}$, STAT-3, PI3K/Akt, AP-1 and other signaling molecules. As a consequence, Dz1 blocks cell proliferation, invasion and metastasis and promotes cell apoptosis [7]. Furthermore, animal tests demonstrated that Dz1 blocks the solid tumor's growth by down-regulating the expression level of LMPI in vivo and enhances the radiosensitivity of NPC [8]. 


\subsection{Other applications of DNAzymes associated with cancer}

DNA methylation, catalyzed by the enzyme DNA methyltransferase (DNMT), typically occurs at CpG sites (cytosine-phosphate-guanine sites) in vertebrates. This pattern of methylation has recently become an important topic for cancer research. Neoplasia is characterized by "methylation imbalance" where genome-wide hypo-methylation is accompanied by localized hypermethylation and increased expression of DNA methyltransferase [29]. The overall methylation state in a cell might also be a precipitating factor in carcinogenesis as evidence suggests that genomewide hypo-methylation can lead to chromosome instability and increase mutation rates. The methylation state of some genes can be used as a biomarker for tumorigenesis [30]. Recently, based on the identification of DNMT and methylation-sensitive endonucleases, Li et al. [31] designed a simple, colorimetric and label-free methylation-responsive DNAzyme (MR-DNAzyme) that detects DNMT activity. This group demonstrated that this strategy for detecting DNMT activity could be used in two ways: as a DNAzymehairpin hybrid probe and in a methylation-responsive DNAbased machine. The MR-DNAzyme could also be used to conveniently and efficiently monitor the process of DNA methylation catalyzed by DNMT. When compared with the traditional method to detect DNA methylation, these DNAzyme-based methods have many advantages: they are less expensive, easier to synthesize and have a higher sensitivity.

\section{Problems and questions on the applications of DNAzymes}

Even though DNAzymes can effectively bind to and cleave target mRNA in vitro and in vivo, their biological effects are easily influenced by many factors, such as the serum stability and the delivery strategy. Studies have shown that certain chemical modifications can greatly increase the DNAzymes' stability in serum, enhance their target binding affinity and extend their half-life. These modifications include phosphorothioate (PS) linkages, $3^{\prime}-3^{\prime}$ inverted nucleotides at the $3^{\prime}$ end of the DNAzyme and locked nucleic acids (LNAs). However, the introduction of PS linkages may affect cleavage efficiency and has been associated with toxicity, immunological responsiveness and nonspecific reactions. So more innovative chemical modifications are needed to strengthen the stability of DNAzymes. A further attempt is the introduction of hairpin DNAzymes, which are created by the addition of stem-loop hairpins to the end of the substratebinding arms of the DNAzyme. While hairpin DNAzymes display resistance to nucleolytic degradation and produce better gene intervention, they do not induce nonspecific effects or cytotoxicity [2,3,32].
As in all nucleic acid-based reagents, delivery is an important issue. Drug delivery systems (DDSs) can help to solve the problems and questions about DNAzymes' stability, biological effects and toxicity. Several seminal studies have demonstrated that certain DNAzyme delivery systems can efficiently encapsulate DNAzymes and transfect them into cells without clear toxicity. The first delivery system used microspheres of the co-polymers poly(lactic acid) and poly (glycolic acid) (PLGA), which encapsulated the DNAzymes inside. PLGA microspheres were able to achieve sustained release and accumulation of the DNAzyme. In a second delivery system, DNAzymes were incorporated into transferrin-modified PEGylated polyplexes (Tf-PEG-polyplex) and administered intraperitoneally or intravenously. Whole body imaging (WBI) revealed that the tumors showed high fluorescence and a large concentration of DNAzymes. However, transferrin (Tf) might aid in targeting DNAzymes to blood-rich organs, which may induce side effects in these organs. A third delivery system used synthetic macromolecules with a branched molecular structure called dendrimers. They can bind the DNAzymes better and facilitate DNAzymes release. So the displayed transfection efficiency can be achieved at lower change ratios of DNAzyme: dendrimer as compared to other tranfection reagents above mentioned [33]. Finally, nanoparticulate systems could enhance the efficacy of tumoricidal DNAzymes with no toxicity and no side effects. DNAzymes delivered in this manner significantly inhibit tumor growth [34]. These results indicate that with more suitable delivery systems, the biological effects of DNAzymes would be further increased, and the DNAzymes could be applied to new subject areas.

\section{Concluding remarks}

Small-molecule nucleic acid technology is now playing an important role for the development of gene-targeted therapy. New discoveries about nucleic acid have enhanced our knowledge of DNA. DNAzymes, first synthesized experimentally in 1994 by Breaker and Joyce, are single-stranded DNA catalysts that can cleave the mRNA of targeted genes effectively and specifically.

Recently, gene targeting has entered a new era with the development of potent and effective gene intervention techniques that act on mRNA. In particular, DNAzymes, ribozymes, siRNA and ASO have been used to treat diseases, including cancer [5]. Among these methods, DNAzymes have potentially broader uses in biology, including detecting specific genes and knocking down pathophysiologically active genes.

From their early use as tools for molecular dissection, DNAzymes are now moving toward clinical applications [35]. Since the activity of DNAzymes was first demonstrated in vivo in 1999, many preclinical studies using animal tumor models have indicated that DNAzymes may 
have clinical utility as inhibitory agents [36]. Characteristics of DNAzymes, such as their enhanced stability and lack of side effects, as well as improved delivery vehicles, have paved the way for DNAzymes to be studied in clinical trials [37]. If clinical tests show safety and tolerability, DNAzymes may be approved for a much greater range of applications. Moreover, with the development of improved DDSs, the biological effects of DNAzymes could be enhanced. Although the development of DNAzymes as drugs is still in its infancy, these agents may become economical and practical anti-cancer therapies in the near future.

This work was supported by the National Natural Science Foundation of China (30873010 and 81072220) and the National High Technology Research and Development Program of China (2009AA02Z403).

1 Breaker R R, Joyce G F. A DNA enzyme that cleaves RNA. Chem Biol, 1994, 1: 223-229

2 Baum D A, Silverman S K. Deoxyribozymes: Useful DNA catalysts in vitro and in vivo. Cell Mol Life Sci, 2008, 65: 2156-2174

3 Santoro S W, Joyce G F. A general purpose RNA-cleaving DNA enzyme. Proc Natl Acad Sci USA, 1997, 94: 4262-4266

4 Dass C R, Choong P F M. Sequence-related off-target effect of Dz13 against human tumor cells and safety in adult and fetal mice following systemic administration. Oligonucleotides, 2010, 20: 51-60

5 Bhindi R, Fahmy R G, Lowe H C, et al. Brothers in arms: DNA enzymes, short interfering RNA, and the emerging wave of small-molecule nucleic acid-based gene-silencing strategies. Am J Pathol, 2007, 171: 1079-1088

6 Pecot C V, Calin G A, Coleman R L, et al. RNA interference in the clinic: Challenges and future directions. Nat Rev Cancer, 2011, 11: 59-67

7 Yang L F, Lu Z X, Ma X Q, et al. A therapeutic approach to nasopharyngeal carcinomas by DNAzymes targeting EBV LMP-1 gene. Molecules, 2010, 15: 6127-6139

$8 \mathrm{Lu} \mathrm{Z} \mathrm{X,} \mathrm{Ma} \mathrm{X} \mathrm{Q,} \mathrm{Yang} \mathrm{L} \mathrm{F,} \mathrm{et} \mathrm{al.} \mathrm{DNAzymes} \mathrm{targeted} \mathrm{to} \mathrm{EBV-}$ encoded latent membrane protein-1 induce apoptosis and enhance radiosensitivity in nasopharyngeal carcinoma. Cancer Lett, 2008, 265: 226-238

9 Mitchell A, Crispin R D, Sun L Q, et al. Inhibition of human breast carcinoma proliferation, migration, chemoinvasion and solid tumor growth by DNAzymes targeting the zinc finger transcription factor EGR-1. Nucleic Acids Res, 2004, 32: 3065-3069

10 Zeng W Z, Deng L Y, Zhou R. Experimental study of targeting $M M P-9$ deoxyribozyme role of adhesion and migration in human lung adenocarcinoma cancer cell (in Chinese). Chin J Lung Cancer, 2008, 11: 765-768

11 Yang L F, Zeng W Z, Li D, et al. Inhibition of cell proliferation, migration and invasion by DNAzyme targeting MMP-9 in A549 cells. Oncol Rep, 2009, 22: 121-126

12 Min Z, Zhao H, Luo F Y, et al. IGF- II inhibitory DNAzymes inhibit the invasion and migration of hepatocarcinoma cells. Biotechnol Lett, 2011, 33: 911-917

13 Liang Z Y, Wei S Z, Guan J, et al. DNAzyme-mediated cleavage of survivin mRNA and inhibition of the growth of PANC-1 cells. J Gastroen Hepatol, 2005, 20: 1595-1602

14 Wiktorska M, Papiewska-Pajak L, Okruszek A, et al. DNAzyme as an efficient tool to modulate invasiveness of human carcinoma cells. Acta Biochim Pol, 2010, 57: 269-275

15 Choi B R, Gwak J, Kwon H M, et al. Oligodeoxyribozymes that cleave $\beta$-catenin messenger RNA inhibit growth of colon cells via reduction of $\beta$-catenin response transcription. Mol Cancer Ther, 2010, 9: 1894-1902

16 Yu S H, Wang T H, Au L C. Specific repression of mutant $K-R A S$ by 10-23 DNAzyme: Sensitizing cancer cell to anti-cancer therapies. Biochem Biophys Res Commun, 2009, 378: 230-234

17 Qu Y, Zhang L, Mao M, et al. Effects of DNAzymes targeting aurora kinase A on the growth of human prostate cancer. Cancer Gene Ther, 2008, 15: 517-525

18 Zhang G, Luo X, Sumithran E, et al. Squamous cell carcinoma growth in mice and in culture is regulated by $c$-Jun and its control of matrix metalloproteinase-2 and -9 expression. Oncogene, 2006, 25: 7260-7266

19 Wu Y P, Yu L J, Mcmahon R, et al. Inhibition of $b c r-a b l$ oncogene expression by novel deoxyribozymes (DNAzymes). Hum Gene Ther, 1999, 10: 2847-2857

20 Kabuli M, Yin J A L, Tobal K. Targeting PML/RAR $\alpha$ transcript with DNAzymes results in reduction of proliferation and induction of apoptosis in APL cells. Hematol J, 2004, 5: 426-433

21 Dass C R, Galloway S J, Jonathan C M, et al. Involvement of $c$-jun in human liposarcoma growth: Supporting data from clinical immunohistochemistry and DNAzyme efficacy. Cancer Biol Ther, 2008, 7: 1297-1301

22 de Bock C E, Lin Z, Itoh T, et al. Inhibition of urokinase receptor gene expression and cell invasion by anti- $u P A R$ DNAzymes in osteosarcoma cells. FEBS J, 2005, 272: 3572-3582

23 Wang Y F, Shen J N, Shang X F, et al. Ezrin mRNA target site selection for DNAzymes using secondary structure and hybridization thermodynamics. Tumor Biol, 2011, 32: 809-817

24 Dass C R, Galloway S J, Choong P F M. Dz13, a c-Jun DNAzyme, is a potent inducer of caspase-2 activation. Oligonucleotides, 2010, 20: 137-145

25 Quaranta V. Motility cues in the tumor microenvironment. Differentiation, 2002, 70: 590-598

26 Stec M J, Niewiarowska J, Nawrot M, et al. DNAzyme to $\beta 1$ and $\beta 3$ mRNA down-regulate expression of the targeted integrins and inhibit endothelial cell capillary tube formation in fibrin and matrigel. J Biol Chem, 2002, 277: 6779-6787

27 Zhang L, Gasper W J, Sanford A, et al. Angiogenic inhibition mediated by a DNAzyme that targets vascular endothelial growth factor receptor 2. Cancer Res, 2002, 62: 5463-5469

28 Zhang G S, Dass C R, Sun L Q, et al. Effect of deoxyribozymes targeting $c$-Jun on solid tumor growth and angiogenesis in rodents. J Natl Cancer Inst, 2004, 96: 683-696

29 Baylin S B, Herman J G, Graff J R, et al. Alterations in DNA methylation: A fundamental aspect of neoplasia. Adv Cancer Res, 1998, 72: $141-196$

30 Brueckner B, Lyko F. DNA methyltransferase inhibitors: Old and new drugs for an epigenetic cancer therapy. Trends Pharmacol Sci, 2004, 25: 551-554

31 Li W, Liu Z L, Yao S Z, et al. Label-free colorimetric assay for methyl-transferase activity based on a novel methylation-responsive DNAzyme strategy. Anal Chem, 2010, 82: 1935-1941

32 Benson V L, Khachigian L M, Lowe H C. DNAzymes and cardiovascular disease. Brit J Pharmacol, 2008, 154: 741-748

33 Tan M L, Choong P F M, Dass C R. DNAzyme delivery systems: Getting past first base. Expert Opin Drug Del, 2009, 6: 127-138

34 Tan M L, Dunstan D E, Friedhuber A M, et al. A nanoparticulate system that enhances the efficacy of the tumoricide Dz13 when administered proximal to the lesion site. J Control Release, 2010, 144: 196-202

35 Dass C R, Choong P F M, Khachigian L M. DNAzyme technology and cancer therapy: Cleave and let die. Mol Cancer Ther, 2008, 7: 243-251

36 Chan C W S, Khachigian L M. DNAzymes and their therapeutic possibilities. Intern Med J, 2009, 39: 249-251

37 Dass C R. Deoxyribozymes: Cleaving a path to clinical trials. Trends Pharmacol Sci, 2004, 25: 395-397

Open Access This article is distributed under the terms of the Creative Commons Attribution License which permits any use, distribution, and reproduction in any medium, provided the original author(s) and source are credited. 